\title{
Effect of tranexamic acid on mortality in patients with traumatic bleeding: prespecified analysis of data from randomised controlled trial
}

\author{
@() $\Theta$ OPEN ACCESS
}

\author{
Ian Roberts professor ${ }^{1}$, Pablo Perel senior clinical lecturer ${ }^{1}$, David Prieto-Merino lecturer, medical \\ statistics ${ }^{1}$, Haleema Shakur senior lecturer in clinical trials ${ }^{1}$, Tim Coats professor ${ }^{2}$, Beverley J Hunt \\ professor $^{3}$, Fiona Lecky professor ${ }^{4}$, Karim Brohi professor ${ }^{5}$, Keith Willett professor ${ }^{6}$, on behalf of \\ the CRASH-2 collaborators.
}

${ }^{1}$ London School of Hygiene and Tropical Medicine, London WC1E 7HT, UK; ${ }^{2}$ Department of Cardiovascular Sciences, University of Leicester, Leicester LE1 5WW, UK; ${ }^{3}$ Guy's and St Thomas' NHS Foundation Trust, London SE1 7EH; ${ }^{4}$ School of Health and Related Research, University of Sheffield, Sheffield S1 4DA, UK; ${ }^{5}$ Centre for Trauma Sciences, Barts and The London School of Medicine and Dentistry, Queen Mary University of London, London; ${ }^{6}$ Nuffield Department of Orthopaedics, Rheumatology and Musculoskeletal Sciences, University of Oxford, Nuffield Orthopaedic Centre, Oxford OX3 7HE, UK

\begin{abstract}
Objectives To examine whether the effect of tranexamic acid on the risk of death and thrombotic events in patients with traumatic bleeding varies according to baseline risk of death. To assess the extent to which current protocols for treatment with tranexamic acid maximise benefits to patients.

Design Prespecified stratified analysis of data from an international multicentre randomised controlled trial (the CRASH-2 trial) with an estimation of the proportion of premature deaths that could potentially be averted through the administration of tranexamic acid.

Participants 13273 trauma patients in the CRASH-2 trial who were treated with tranexamic acid or placebo within three hours of injury and trauma patients enrolled in UK Trauma and Audit Research Network, stratified by risk of death at baseline $(<6 \%, 6-20 \%, 21-50 \%,>50 \%)$.

Intervention Tranexamic acid (1 g over 10 minutes followed by $1 \mathrm{~g}$ over eight hours) or matching placebo.

Main outcome measure Odds ratios and 95\% confidence intervals for death in hospital within four weeks of injury, deaths from bleeding, and fatal and non-fatal thrombotic events associated with the use of tranexamic acid according to baseline risk of death. Unless there was strong evidence against the null hypothesis of homogeneity of effects $(\mathrm{P}<0.001)$, the overall odds ratio was used as the most reliable guide to the odds ratios in all strata.

Results Tranexamic acid was associated with a significant reduction in all cause mortality and deaths from bleeding. In each stratum of baseline risk, there were fewer deaths among patients treated with tranexamic acid. There was no evidence of heterogeneity in the effect of tranexamic acid on all cause mortality ( $\mathrm{P}=0.96$ for interaction) or deaths from
\end{abstract}

bleeding $(\mathrm{P}=0.98)$ by baseline risk of death. In those treated with tranexamic acid there was a significant reduction in the odds of fatal and non-fatal thrombotic events (odds ratio $0.69,95 \%$ confidence interval 0.53 to $0.89 ; \mathrm{P}=0.005$ ) and a significant reduction in arterial thrombotic events $(0.58,0.40$ to $0.83 ; P=0.003)$ but no significant reduction in venous thrombotic events $(0.83,0.59$ to $1.17 ; P=0.295)$. There was no evidence of heterogeneity in the effect of tranexamic acid on the risk of thrombotic events $(P=0.74)$. If the effect of tranexamic acid is assumed to be the same in all risk strata $(<6 \%, 6-20 \%, 21-50 \%,>50 \%$ risk of death at baseline), the percentage of deaths that could be averted by administration of tranexamic acid within three hours of injury in each group is $17 \%, 36 \%, 30 \%$, and $17 \%$, respectively.

Conclusions Tranexamic acid can be administered safely to a wide spectrum of patients with traumatic bleeding and should not be restricted to the most severely injured.

Trial registration ISRCTN86750102.

\section{Introduction}

The CRASH-2 trial showed that a short course of tranexamic acid given within three hours of injury to adult patients with traumatic bleeding significantly reduces all cause mortality with no apparent increase in the risk of adverse thrombotic events. ${ }^{12}$ As a result, tranexamic acid is now being incorporated into trauma protocols around the world. ${ }^{3-5}$ These protocols generally focus on the care of the most severely injured. Patients with a high risk of death at baseline have the most to gain from the use of life saving treatments because the absolute benefits of an effective treatment tend to increase as the baseline risk increases. On the other hand, there are more low risk trauma patients than 
high risk patients, and a large number of patients at low risk might contribute more deaths than a smaller number of patients at high risk. We carried out prespecified analyses of the CRASH-2 trial data to examine how effects of treatment with tranexamic acid vary according to the baseline risk of death in patients with traumatic bleeding. We then used data from a large hospital based trauma registry to assess the extent to which current trauma protocols maximise the benefits to patients of treatment with tranexamic acid.

\section{Methods}

We have previously used data from the CRASH-2 trial to develop a prognostic model to identify patients with life threatening traumatic bleeding. ${ }^{126}$ The model predicts all cause mortality and includes the following baseline variables: age, score on Glasgow coma scale, systolic blood pressure, heart rate, respiratory rate, hours since injury, and type of injury. We validated the model in a large sample of trauma patients from the UK Trauma and Audit Research Network and found that discrimination (C statistic above 0.80 ) and calibration were good. A full description of derivation and validation of the model has been published elsewhere. ${ }^{6}$

For these analyses, which were prespecified in the planned statistical analysis, we used the prognostic model to stratify patients in the CRASH-2 trial whose treatment was initiated within three hours after injury into four strata of risk of mortality $(<6 \%, 6-20 \%, 21-50 \%,>50 \%)$. These strata were chosen because we previously developed a simple risk chart to classify patients into these risk categories. ${ }^{6}$ We then examined the effect of tranexamic acid ( $1 \mathrm{~g}$ over 10 minutes followed by $1 \mathrm{~g}$ over eight hours) on all cause mortality, deaths from bleeding, and thrombotic events (fatal and non-fatal myocardial infarction, stroke, deep vein thrombosis, and pulmonary embolism) within these strata. We used $\chi^{2}$ to assess heterogeneity in treatment effects across subgroups. When subgroup effects are examined, it is important to consider the possibility that an observed subgroup effect might have arisen by chance alone. As recommended in published guidelines on subgroup analysis, to reduce the likelihood of making inappropriate subgroup inferences we prespecified that unless there was strong evidence against the null hypothesis of homogeneity of effects (that is, $\mathrm{P}<0.001$ ), the overall odds ratio would be considered the most reliable guide to the approximate odds ratio in all strata. ${ }^{7} \mathrm{We}$ examined the effect of tranexamic acid on arterial (myocardial infarction and stroke) and venous (deep vein thrombosis and pulmonary embolism) thrombotic events combined and separately.

We also used the prognostic model to estimate the number and proportion of deaths at different levels of baseline risk in UK trauma patients using data from the UK Trauma Audit and Research Network. This analysis was conducted to assess the distribution of risk of death in a representative trauma population. The network dataset includes data from $60 \%$ of hospitals receiving trauma patients in England and Wales. ${ }^{8}$ Data are collected on patients who meet any of the following criteria: death from injury at any point during admission, hospital stay longer than three days, requirement for intensive or high dependency care, requirement for transfer between hospitals for specialist care. For these analyses, we included patients presenting between 2000 and 2008 to hospitals participating in the UK Trauma Audit and Research Network with an estimated blood loss of at least $20 \%$ on the basis that these patients can be considered to have severe haemorrhage. Estimated blood loss was calculated in patients in the network by allocating an estimated percentage of total volume of blood lost for each injury code in the Abbreviated Injury Scale (AIS) dictionary by blinded, then consensus, agreement from two emergency physicians, as described in detail elsewhere. ${ }^{9}$ Finally, for each patient we used the prognostic model described above to estimate the predicted risk of death and then estimated the proportion of premature deaths averted by applying the odds ratio from the CRASH-2 trial to each of the risk strata.

\section{Results}

Table $1 \Downarrow$ shows the characteristics of patients in the CRASH-2 trial and the UK Trauma Audit and Research Network according to risk of death at baseline. Patients with a higher baseline risk were older, had a lower systolic blood pressure, had a lower score on the Glasgow coma scale, had a higher heart and respiratory rate, and were more likely to have trauma from a blunt injury than patients with a lower risk. Most CRASH-2 trial patients $(97 \%)$ were from middle income countries, and this frequency distribution was similar for all the risk strata.

Figure $1 \Downarrow$ shows the effect of tranexamic acid on all cause mortality by risk of death at baseline. In each stratum of baseline risk, there were fewer deaths among patients treated with tranexamic acid, although in the lowest risk strata, the effect was not significant. There was no evidence of heterogeneity in the effect of tranexamic acid on all cause mortality $(\mathrm{P}=0.96$ for interaction).

Figures $2 \Downarrow$ and $3 \Downarrow$ show the effect of tranexamic acid on death from bleeding and on fatal and non-fatal thrombotic events by baseline risk of death. There was no evidence of heterogeneity in the effect of tranexamic acid on deaths from bleeding $(\mathrm{P}=0.98)$ or on thrombotic events $(\mathrm{P}=0.74)$. There was no significant reduction in all other causes of death combined (that is, non-bleeding deaths) (odds ratio $0.97,95 \%$ confidence interval 0.86 to 1.09 ) and no evidence of heterogeneity according to baseline risk $(\mathrm{P}=0.99)$.

Table $2 \Downarrow$ shows the effect of tranexamic acid on fatal and non-fatal thrombotic events in patients treated within three hours of injury. In patients treated with tranexamic acid there was a significant reduction in the risk of fatal and non-fatal thrombotic events (odds ratio $0.69,95 \%$ confidence interval 0.53 to 0.89 ; $\mathrm{P}=0.005)$ and a significant reduction in arterial thrombotic events $(0.58,0.40$ to $0.83 ; \mathrm{P}=0.003)$. There was no evidence of heterogeneity in the effect of tranexamic acid on arterial thrombotic events $(\mathrm{P}=0.91)$ or venous thrombotic events by baseline risk $(\mathrm{P}=0.85)$. There was also no significant reduction in the risk of venous thrombotic events with tranexamic acid $(0.83,0.59$ to $1.17 ; \mathrm{P}=0.295)$.

Figure $4 \Downarrow$ shows the distribution of patients in the UK Trauma Audit and Research Network by baseline risk. There were many more low risk patients than high risk patients. If we assume that the effect of tranexamic acid is the same in all risk strata (constant odds ratio), the estimated overall percentage of deaths that could be avoided by administering tranexamic acid, split according to each risk stratum $(<6 \%, 6-20 \%, 21-50 \%,>50 \%)$ is $17 \%, 36 \%, 30 \%$, and $17 \%$, respectively. Figure $5 \Downarrow$ shows the number of deaths that could potentially be averted through the use of tranexamic acid in patients in the UK Trauma Audit and Research Network by baseline risk of death.

\section{Discussion}

The beneficial effects of tranexamic acid in reducing all cause mortality and deaths from bleeding in patients with traumatic bleeding do not seem to vary significantly by baseline risk of 
death. Tranexamic acid reduced the odds of death from bleeding by about $30 \%$ in each of the baseline risk strata studied and reduced the odds of thrombotic events by about $30 \%$. Once again, this reduction did not vary significantly by baseline risk of death. Taken together, these data suggest that tranexamic acid can be administered safely to a wide spectrum of patients with traumatic bleeding and that its use should not be restricted to those with the most severe haemorrhage. Absence of evidence of heterogeneity by baseline risk of death, however, should not be taken as evidence of absence. In particular, in the lowest risk group the precision of the estimated effect is low, and there remains some uncertainty.

\section{Strengths and weaknesses}

The data examined are from a large randomised placebo controlled trial with well concealed allocation, blinded outcome assessment, and minimal loss to follow up. We prespecified this analysis of the effect of tranexamic acid stratified by baseline risk of death in the planned statistical analysis in 2010 before un-blinding. Specifically, we stated that we would conduct "subgroup analyses according to baseline risk of mortality. We will develop a prognostic model including all the baseline variables (age, sex, systolic blood pressure, heart rate, respiratory rate, Glasgow Coma Score, number of hours since injury, central capillary refill time and type of injury). This model will be derived only from the control group. We will categorise the patients according to tertiles of risk and we will report the relative and absolute measures of effect for each category." The only substantial deviation from this plan was that we created four risk strata rather than three. This was done so that the risk groups analysed corresponded with those in a risk score that we had previously developed for use by emergency physicians. ${ }^{6}$ The risk score categories in turn were based on previous research and the views of clinical users. ${ }^{6}$

The data examined here are a subset of the entire CRASH-2 trial. As most trauma protocols restrict use of tranexamic acid to patients who are within three hours of injury, we also restricted our analyses to these patients. Given that our results are based on a subgroup analysis, they should be interpreted cautiously. On the other hand, there was also a reduction in the risk of fatal and non-fatal myocardial infarction and fewer thrombotic events with tranexamic acid (relative risk 0.64, 95\% confidence interval 0.42 to $0.97 ; \mathrm{P}=0.035$ ) in the main trial analysis of patients treated up to eight hours after injury. ${ }^{1}$ While there could be grounds for scepticism about the reduction in arterial thrombotic events with tranexamic acid, the data are reassuring that there is no increased risk. As regards the absence of evidence of an interaction between the effect of tranexamic acid and baseline risk of death, it is important to bear in mind that this could be because of a lack of statistical power for the interaction tests.

That we analysed data from a randomised controlled trial using a prognostic model that was derived from the same trial might be considered a weakness. On the other hand, the prognostic model we used has good external calibration and discrimination, and there are few models available that can be reliably applied to the trauma population included in the CRASH-2 trial. The revised trauma score, one of the most widely used scores, was developed many years ago, has several methodological limitations, and showed poor calibration in the CRASH-2 and UK Trauma Audit and Research Network datasets (data available on request).

Establishing cause of death in trauma patients can be difficult, and any inaccuracy might have affected our estimate of the effect of tranexamic acid on fatal thrombotic events. There might also have been inaccuracy in the diagnosis of non-fatal events. The diagnosis of myocardial infarction is particularly challenging in trauma patients, many of whom are anaesthetised or sedated. Increases in creatine kinase MB isoenzyme activity are hard to interpret because of muscle injury, and we did not collect data on troponin. Diagnostic inaccuracy, however, tends to obscure treatment effects and would not readily explain the observed reduction in thrombotic events with tranexamic acid.

\section{Potential biological mechanisms}

Tranexamic acid reduces the risk of death from haemorrhage and seems to reduce the risk of arterial thrombotic events. It is possible that by reducing bleeding, tranexamic acid reduces myocardial oxygen demand and increases oxygen supply. It is also possible that the reduction in arterial thrombotic events could be mediated through an anti-inflammatory effect of tranexamic acid. ${ }^{10} 11$

\section{Implications for patients}

These results have important implications for the care of trauma patients. Firstly, they suggest that tranexamic acid can safely be used in all trauma patients with or at risk of severe bleeding, as in the inclusion criteria used in the CRASH-2 trial, and not just in the most severely injured. Secondly, the observed reduction in the risk of arterial events with tranexamic acid suggests that the absolute benefits from administration of tranexamic acid are likely to be greatest in older trauma patients, who at any given level of severity of injury have a higher baseline risk of death from haemorrhage and thrombotic events. ${ }^{6}{ }^{12}$ Clinical concern about an increased risk of ischaemic cardiac events might be a reason to give rather than to withhold tranexamic acid. It is worth noting that trials of tranexamic acid in patients undergoing cardiac surgery, a group at high risk of cardiac events, provide no evidence of any increased risk. ${ }^{13} \mathrm{We}$ acknowledge that estimating the risk of severe bleeding is a challenging ongoing process that uses not only physiological variables but other variables such as laboratory measurements and response to treatments. Physicians will use all this information and their clinical judgment when deciding whether to use tranexamic acid. In situations of uncertainty, however, physicians can use the prognostic model to support the decision making process and should certainly consider administering tranexamic acid to patients with a risk of death higher than 5\%.

We thank the CRASH-2 Trial Collaborators and the UK Trauma Audit and Research Network Executive for making their data available. PP and IR are members of the Medical Research Council Prognosis Research Strategy (PROGRESS) Partnership (G0902393/99558).

Contributors: IR, PP, DP-M and HS proposed the study. DP-M conducted the analyses. All authors contributed to interpreting the data and drafting the manuscript. IR is guarantor.

Funding: This study was funded by the UK Health Technology Assessment programme (09/22/165) and will be published in full in the Health Technology Assessment journal series. The views and opinions expressed therein are those of the authors and do not necessarily reflect those of the Department of Health.

Competing interests: All authors have completed the ICMJE uniform disclosure form at www.icmje.org/coi_disclosure.pdf (available on request from the corresponding author) and declare that the London School of Hygiene and Tropical Medicine has received funding from Pfizer, who manufacture tranexamic acid.

Ethical approval: The London School of Hygiene and Tropical gave ethical approval for this study and the use of the CRASH-2 trial data. 


\section{What is already known on this topic}

Tranexamic acid given to patients with traumatic bleeding within three hours of injury significantly reduces mortality with no apparent increase in adverse thrombotic events

\section{What this study adds}

The beneficial effect of tranexamic acid on all cause mortality or deaths from bleeding is not affected by baseline risk of death There were fewer thrombotic events with tranexamic acid with no evidence of heterogeneity by baseline risk Use of tranexamic acid should not be limited to high risk patients

TARN already has ethical approval (PIAG section 60) for research on the anonymised data that are stored securely on the University of Manchester server.

Data sharing: The CRASH-2 trial data are publicly available via an online data sharing website called FREEBIRD that can be accessed at https: //ctu2.Ishtm.ac.uk/freebird/. Unpublished calibration data available from the corresponding author. Consent was not obtained for sharing data but the presented data are anonymised and risk of identification is low.

1 CRASH-2 Collaborators. Effects of tranexamic acid on death, vascular occlusive events, and blood transfusion in trauma patients with significant haemorrhage (CRASH-2): a randomised, placebo-controlled trial. Lancet 2010;376:23-32.

2 CRASH-2 collaborators. The importance of early treatment with tranexamic acid in bleeding trauma patients: an exploratory analysis of the CRASH-2 randomised controlled trial. Lancet 2011;377:1096-101.

3 TXA implementation pages-how to do it. www2.le.ac.uk/departments/cardiovascular sciences/research/population-research-and-clinical-trials/emergency-medicine-group/ research/injury/txa-implementation-pages-how-to-do-it.

4 Committee on Tactical Combat Casualty Care. Tranexamic acid (TXA) in tactical combat casualty care. Guideline revision recommendation. 2011. www.medicalsci.com/files/ tranexamic_acid_txa_in_tactical_combat_casualty_care.pdf.

5 Luz L, Sankarankutty A, Passos E, Rizoli S, Fraga G, Nascimento Jr B. Tranexamic acid for traumatic hemorrhage. Rev Col Bras Cir. 2012:39:77-80.
6 Perel P, Prieto-Merino D, Shakur H, Clayton T, Lecky F, Bouamra O, et al. Predicting early death in patients with traumatic bleeding: development and validation of prognostic model. BMJ 2012;345:e5166.

7 Sun X, Briel M, Walter SD, Guyatt GH. Is a subgroup effect believable? Updating criteria to evaluate the credibility of subgroup analyses. BMJ 2010;340:c117.

8 Trauma Audit and Research Network. www.tarn.ac.uk/.

9 Guly HR, Bouamra O, Hatton M, Dark P, Coats T, Driscoll P, et al. Vital signs and estimated blood loss in patients with major trauma: testing the validity of the ATLS classification of hypovolaemic shock. Resuscitation 2011;82:556-9.

10 Godier A, Roberts I, Hunt B. Tranexamic acid: less bleeding and less thrombosis. Crit Care 2012;16:135.

11 Jiménez J, Iribarren J, Lorente L, Rodríguez J, Hernandez D, Nassar I, et al. Tranexamic acid attenuates inflammatory response in cardiopulmonary bypass surgery through blockade of fibrinolysis: a case control study followed by a randomized double-blind controlled trial. Crit Care 2007;11:R117.

12 Geerts WH, Code KI, Jay RM, Chen E, Szalai JP. A prospective study of venous thromboembolism after major trauma. N Engl J Med 1994;331:1601-6.

13 Ker K, Edwards $\mathrm{P}$, Perel $\mathrm{P}$, Shakur $\mathrm{H}$, Roberts I. Effect of tranexamic acid on surgical bleeding: systematic review and cumulative meta-analysis. BMJ 2012;344:e3054.

Accepted: 18 August 2012

\section{Cite this as: BMJ 2012;345:e5839}

This is an open-access article distributed under the terms of the Creative Commons Attribution Non-commercial License, which permits use, distribution, and reproduction in any medium, provided the original work is properly cited, the use is non commercial and is otherwise in compliance with the license. See: http://creativecommons.org/licenses/by$\mathrm{nc} / 2.0 /$ and http://creativecommons.org/licenses/by-nc/2.0/legalcode. 


\section{Tables}

Table 1/ Characteristics of patients with traumatic bleeding in CRASH-2 and Trauma Audit and Research Network (TARN) by risk of death at baseline. Figures are numbers (percentages) unless stated otherwise

\begin{tabular}{lcccc} 
& \multicolumn{4}{c}{ Baseline risk } \\
\cline { 2 - 5 } CRASH-2 & $\leq 5 \%$ & $6-20 \%$ & $21-50 \%$ & $>50 \%$ \\
\hline No of patients & $4840(36)$ & $5395(41)$ & $1848(14)$ & $1190(9)$ \\
\hline Men & $4111(85)$ & $4457(83)$ & $1533(83)$ & $1002(84)$ \\
\hline Blunt injury & $2761(57)$ & $3186(59)$ & $1316(71)$ & $980(82)$ \\
\hline Mean (IQR) age (years) & $27(22-36)$ & $32(25-44)$ & $35(25-48)$ & $35(25-49)$ \\
\hline Mean (IQR) systolic blood pressure (mm Hg) & $100(90-120)$ & $90(80-110)$ & $80(70-100)$ & $80(60-90)$ \\
\hline Mean (IQR) heart rate (per min) & $100(90-112)$ & $106(92-120)$ & $110(97-124)$ & $118(98-130)$ \\
\hline Mean (IQR) respiratory rate (per min) & $20(18-24)$ & $24(20-28)$ & $24(20-30)$ & $24(16-30)$ \\
\hline Mean (IQR) Glasgow coma score (total) & $15(15-15)$ & $15(13-15)$ & $10(7-13)$ & $4(3-6)$ \\
\hline TARN (Trauma Audit and Research Network) & \multicolumn{3}{c}{} \\
\hline No of patients & $6486(48)$ & $4912(36)$ & $1456(11)$ & $743(6)$ \\
\hline Men & $4954(76)$ & $3239(66)$ & $861(59)$ & $537(72)$ \\
\hline Blunt injury & $5303(82)$ & $4339(88)$ & $1312(90)$ & $716(96)$ \\
\hline Mean (IQR) age (years) & $30(22-41)$ & $52(35-67)$ & $56(31-82)$ & $42(27-67)$ \\
\hline Mean (IQR) systolic blood pressure (mm Hg) & $130(118-142)$ & $126(105-147)$ & $120(96-156)$ & $103(85-130)$ \\
\hline Mean (IQR) heart rate (per min) & $85(75-98)$ & $90(76-108)$ & $100(81-120)$ & $111(94-130)$ \\
\hline Mean (IQR) respiratory rate (per min) & $18(16-22)$ & $20(17-24)$ & $21(18-26)$ & $22(18-27)$ \\
\hline Mean (IQR) Glasgow coma score (total) & $15(15-15)$ & $15(14-15)$ & $10(7-15)$ & $3(3-6)$ \\
\hline
\end{tabular}

IQR=interquartile range. 
Table 2| Effect of tranexamic acid on fatal and non-fatal thrombotic events in patients with traumatic bleeding. Figures are numbers (percentages) of patients experiencing each event

Tranexamic acid $(n=6684)$ Placebo $(n=6589)$ Odds ratio $(95 \% \mathrm{Cl})$ P value

\begin{tabular}{lllll} 
Any event & $98(1.5)$ & $140(2.1)$ & $0.69(0.53$ to 0.89$)$ & 0.005 \\
\hline Any arterial event & $47(0.7)$ & $80(1.2)$ & $0.58(0.40$ to 0.83$)$ & 0.003 \\
\hline Myocardial infarction & $23(0.3)$ & $46(0.7)$ & $0.49(0.30$ to 0.81$)$ & 0.005 \\
\hline Stroke & $28(0.4)$ & $40(0.6)$ & $0.69(0.43$ to 1.11$)$ & 0.128 \\
\hline Any venous event & $60(0.9)$ & $71(1.1)$ & $0.83(0.59$ to 1.17$)$ & 0.295 \\
\hline Pulmonary embolism & $42(0.6)$ & $47(0.7)$ & $0.88(0.58$ to 1.33$)$ & 0.548 \\
\hline Deep vein thrombosis & $25(0.4)$ & $28(0.4)$ & $0.88(0.52$ to 1.50$)$ & 0.641 \\
\hline
\end{tabular}




\section{Figures}

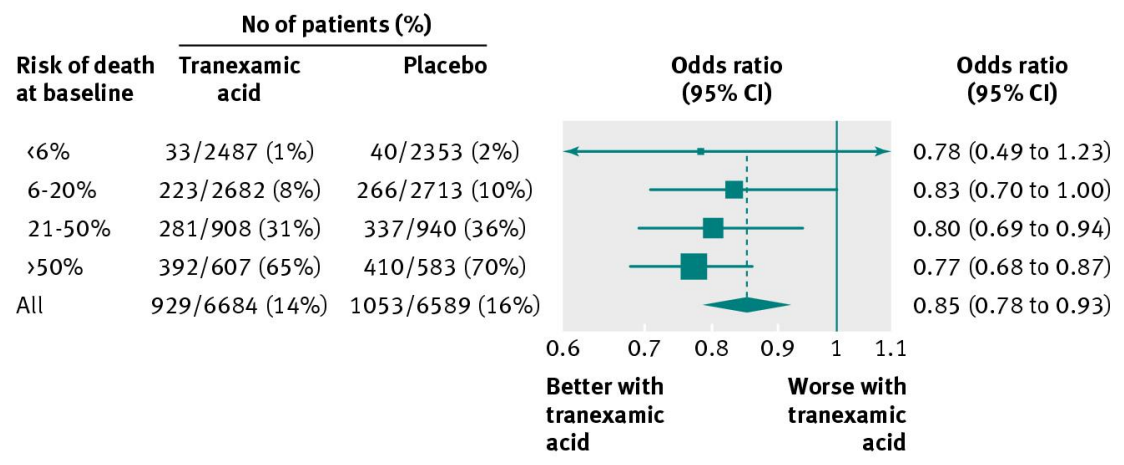

Fig 1 Deaths from all causes in patients with traumatic bleeding according to treatment with tranexamic acid $(P=0.96$ for heterogeneity)

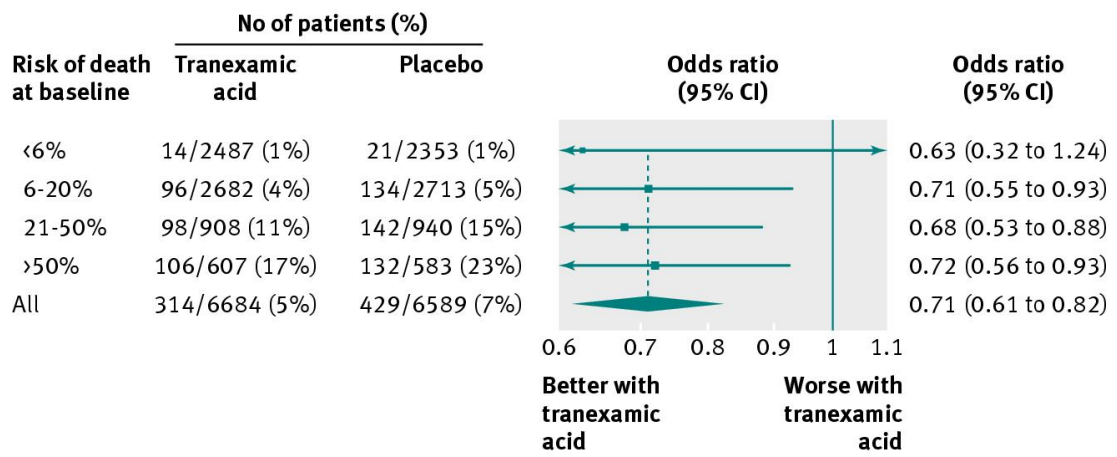

Fig 2 Death from bleeding in patients with traumatic bleeding according to treatment with tranexamic acid ( $P=0.98$ for heterogeneity)

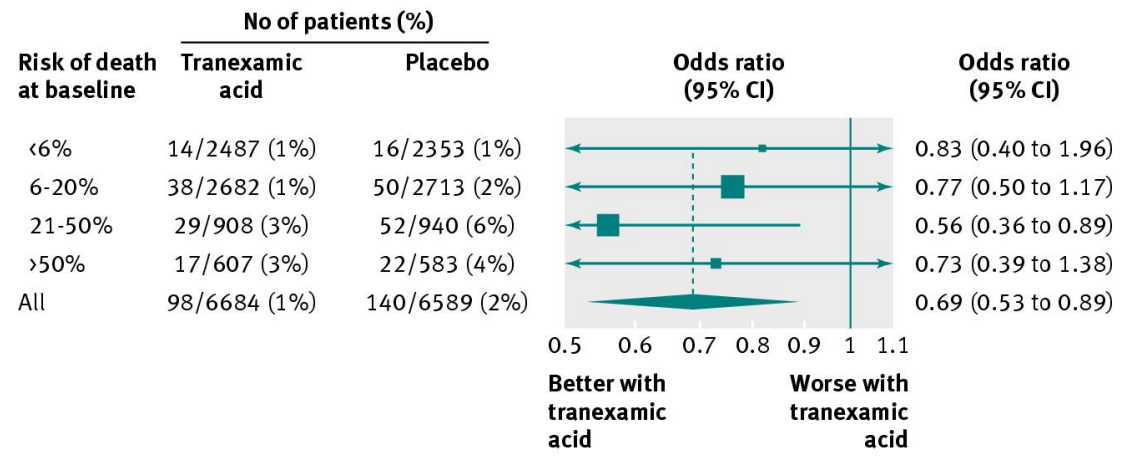

Fig 3 Fatal and non-fatal thrombotic events in patients with traumatic bleeding according to treatment with tranexamic acid ( $P=0.74$ for heterogeneity) 


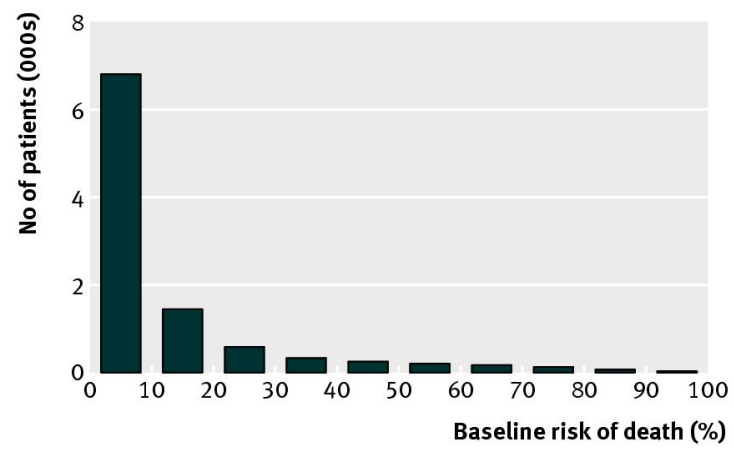

Fig 4 Distribution of patients with traumatic bleeding in UK Trauma Audit and Research Network by baseline risk of death

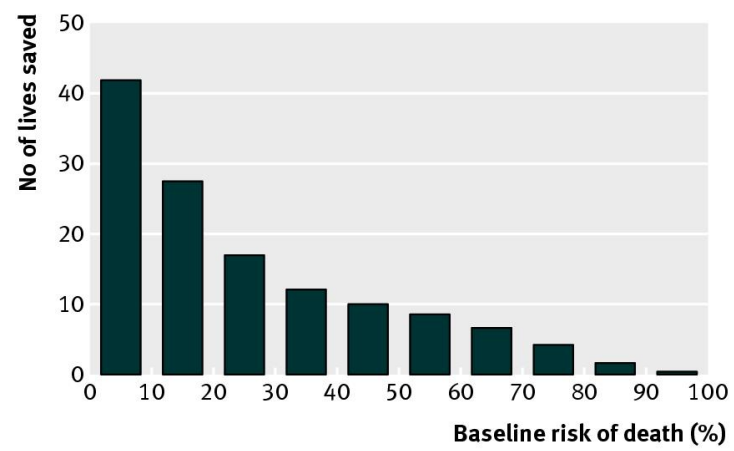

Fig 5 Distribution of estimated premature deaths in patients with traumatic bleeding potentially averted with administration of tranexamic acid 